of various bioactive metabolites. The unique mangrove environment has become the targeted area for the discovery of novel Streptomyces. This 'rainforest of the sea' often experiencing unpredictable environmental changes that could drive species diversity and stimulate the synthesis of unusual bioactive metabolites. This study was aimed to explore the antioxidant and cytotoxic potentials of novel Streptomyces species discovered from the soil of a mangrove forest in Sarawak, Malaysia.

Methods Two novel Streptomyces species were isolated from an under-explored mangrove soil collected at Kuching (Sarawak) and identified using a polyphasic approach. The novel streptomycetes, strains MUSC $1 \mathrm{~J}^{\mathrm{T}}$ and MUSC $93 \mathrm{~J}^{\mathrm{T}}$, were subjected to fermentation and methanol extraction as shown in figure 1(figure 1). The methanolic extracts of both strains were screened for in vitro antioxidant potential and cytotoxic effect against human colon cancer cell lines: HCT-116, HT29, Caco-2, and SW480.

Results Methanolic extracts of Streptomyces monashensis sp. nov. (MUSC $1 \mathrm{~J}^{\mathrm{T}}$ ) and Streptomyces colonosanans sp. nov. (MUSC 93 ${ }^{\mathrm{T}}$ ) exhibited significant antioxidative activity, for instance, MUSC $1 \mathrm{~J}^{\mathrm{T}}$ and MUSC $93 \mathrm{~J}^{\mathrm{T}}$ (at $2 \mathrm{mg} / \mathrm{mL}$ ) exerted $83.80 \pm 4.80 \%$ and $83.32 \pm 2.62 \%$ SOD-like activity respectively. Furthermore, both strains demonstrated promising cytotoxicity against the tested colon cancer cell lines. MUSC $93 \mathrm{~J}^{\mathrm{T}}$ showed the highest cytotoxic activity against SW480 cells; resulting in the lowest cell viability of $63.6 \pm$ $3.0 \%$ after treated with the extract. Extract of MUSC $93 \mathrm{~J}^{\mathrm{T}}$ was also further tested against human normal colon cancer CCD-18Co cells. The result revealed that there is no significant cytotoxic effect of MUSC $93 \mathrm{~J}^{\mathrm{T}}$ extract on CCD-18Co cells.

Conclusions The outcomes of this study demonstrated the anti-colon cancer potential of novel streptomycetes, thus, highlighting the importance of novel strain discovery from underexplored mangrove environment and their role as high-quality resources for chemo-preventive drug discovery.

\section{IDDF2019-ABS-0327 LYSOPHOSPHATIDIC ACID ACTIVATES PI3K/ AKT TO PROMOTES OESOPHAGEAL CANCER}

Si Liu*, Haiyan Jiang, Shengtao Zhu. Department of Gastroenterology, Beijing Friendship Hospital, Capital Medical University, National Clinical Research Center for Digestive Disease, Beijing Digestive Disease Center, Beijing Key Laboratory for Precancerous Lesion of Digestive Disease, Beijing, China

\subsection{6/gutjin-2019-IDDFabstracts.79}

Background Oesophageal cancer (OC) is the eighth most common cancer globally. Lysophosphatidic acid (LPA), a bioactive glycerophospholipid, mediates multiple biological process and diseases, including inflammation and cancer. The expression of autotaxin (ATX), which catalyzes the production of LPA, and LPA receptors are up-regulated in several types of cancer, including ovarian and colon cancer. However, the importance of LPA and LPA receptors in the development and progression of oesophageal cancer is unclear. In this study, we sought to determine whether LPA and LPA receptors regulate the progression of oesophageal cancer.
Methods The expression level of ATX was analyzed in tumor tissues in comparison with adjacent normal tissues of OC patients by immunohistochemistry and the expression level of different LPA receptors $\left(\mathrm{LPA}_{1}-\mathrm{LPA}_{6}\right)$ was determined by RTPCR. Then we examined the potential role of LPA in oesophageal cancer progression by administering $\operatorname{LPA}(5 \mu \mathrm{M})$ to esophageal cancer cell lines KYSE30 and TE-2. Cell proliferation is analyzed by Cell Counting Kit- 8 proliferation assay, EdU labeling and colony formation. Wound-healing assay and transwell assay was used to determine the metastasis of $\mathrm{OC}$ cell lines. Furthermore, we knockdown $\mathrm{LPA}_{1}$ and $\mathrm{LPA}_{2}$ respectively to explore which receptor involved in the effect of LPA. Western Blot was used to detect the signaling pathway activated by LPA.

Results A significant high expression of ATX was found in OC tissues. The expressions of $\mathrm{LPA}_{1}$ and $\mathrm{LPA}_{2}$ were also much higher in KYSE30 and TE-2 compared with HET-1a (normal esophagus epithelial cell lines). Administration of LPA remarkably increased cell proliferation and metastasis in OC cell lines. Furthermore, Knockdown of $\mathrm{LPA}_{1}$ abolished the effect of LPA on promoting oesophageal cancer. In addition, western blot results showed the activation of AKT(p-AKT) was induced by LPA in OC cells. And the PI3K inhibitor LY294002 inhibited both LPA induced AKT activation and cell proliferation in ESCC cells.

Conclusions These results indicated that LPA might regulate the progression of oesophageal cancer by activating PI3K/AKT signaling pathway via $\mathrm{LPA}_{1}$.

\section{IDDF2019-ABS-0339 HIGH-FAT DIET-INDUCED GUT MICROBIOTA DYSBIOSIS ACTIVATE MCP-1/CCR2 PATHWAY AND PROMOTE INTESTINAL CARCINOGENESIS}

Zixuan Guo*, Tianyu Liu, Kui Jiang, Bangmao Wang, Hailong Cao. Department of Gastroenterology and Hepatology, General Hospital, Tianjin Medical University, China

\subsection{6/gutjn|-2019-IDDFabstracts.80}

Background High-fat diet (HFD) is an important risk factor for colorectal cancer (CRC). However, the mechanisms remain to be clarified. The potential pathway MCP-1/CCR2 activation can recruit and polarize M2 tumor-associated macrophages (TAMs). Here we aimed to investigate the effects and mechanisms of HFD on the gut microbiota and intestinal tumor development.

Methods A retrospective cohort study was performed to investigate HFD in relation to advanced colorectal neoplasia (AN). 15 CRC patients from each group for IHC staining were selected to evaluate MCP-1/CCR2 expression. Four-week-old $A p c^{\text {min/+ }}$ mice were randomly divided into HFD group and control group. Intestinal tumor development, cell proliferation and apoptosis, inflammation and MCP-1/CCR2 pathway were determined. Intestinal M2 TAMs were measured by immunofluorescence double staining. Fecal pellets were collected for microbiota and short-chain fatty acids (SCFA) analysis. Antibiotics cocktail was administered with HFD to investigate the effect of crosstalk between the HFD and gut microbiota. Fecal microbiota transplantation (FMT) was used in another batch 
of $A p c^{m i n /+}$ mice to determine the causality between HFDinduced dysbiosis and carcinogenesis.

Results Participants with HFD were more likely to develop AN, especially invasive carcinoma. The expression of MCP-1/ CCR2 and CD163 in CRC patients with HFD was significantly higher. In the $A p c^{\mathrm{min} /+}$ mice, the total number of intestinal adenoma of HFD group was significantly increased. Pathological analysis confirmed intestinal carcinogenesis in small intestine and colon in HFD group, while there were only adenomas in the control group. This was accompanied by promoting tumor cell proliferation and decreasing apoptosis. Moreover, HFD administration altered gut microbiota, with increased opportunistic pathogens and decreased SCFA producing bacteria. The dysbiosis up-regulated the expression of MCP-1 and CCR2. And an increased M2 TAMs was observed in HFD group. And the effects of intestinal carcinogenesis can be restored by antibiotics cocktail. The transfer of fecal microbiota from HFD-fed mice also increased the tumor multiplicity and promoted carcinogenesis, while the MCP-1/ CCR2 axis was also activated.

Conclusions HFD-induced dysbiosis promoted the intestinal carcinogenesis through activating the MCP-1/CCR2 axis.

\section{IDDF2019-ABS-0341 HOMEOBOXC6 PROMOTES METASTASIS OF COLORECTAL CANCER BY ACTIVATING WNT/B-CATENIN AND INDUCING EMT}

Lina Qi*, Wangxiong Hu, Biting Zhou. Zhejiang University, China

\subsection{6/gutjnl-2019-IDDFabstracts.81}

Background HomeoboxC6 (HOXC6) belongs to the homeobox family, members of which encode a highly conserved family of transcription factors that play an important role in morphogenesis in all multicellular organisms. It was found to be upregulated in multiple-cancers, such as breast cancer, prostate cancer, liver cancer, colorectal cancer and so on. Upregulation of HOXC6 also contributed to poor prognosis in colorectal cancer. However, there were very few researches about HOXC6 in colorectal cancer and it was still unknown how it plays its role in tumor cell proliferation and metastasis in colorectal cancer.

Methods

1. Cell culture and HOXC6 overexpressed HCT116 cell line construction

HCT116 was cultured with 1640 medium added with 10\% FBS and 1\% Penicillin-Streptomycin Solution with general culture condition.

2. Clinical patient tumor samples $\underline{Z}$ patients including $\underline{4}$ left-sided patients and $\underline{3}$ right-sided colorectal patients were collected for HOXC6 expression analysis compared to their para-tumor samples

3. Total protein and plasma $\&$ nuclear protein extraction and Western blot analysis

4. CCK8 and transwell with or without matrigel analysis

5. RNASeq and Co-IP analysis

6. Total mRNA extraction and qPCR analysis

7. Survival analysis of gene expression in UALCAN database

Results

1. HOXC6 was upregulated in right-sided colorectal cancer (figure 1A)

2. Upregulation of HOXC6 contributed to migration and invasion but not in proliferation (figure $1 \mathrm{D}, \mathrm{E}$ )

3. Wnt/b-catenin and P53 pathway were activated by HOXC6 upregulation (figure $1 \mathrm{~F}, \mathrm{G}, \mathrm{H}$ )

4. DKK1 was upregulated by HOXC6 and could combined to HOXC6 (figure 1I)

5. EMT was induced by HOXC6 upregulation (figure 1J)
A

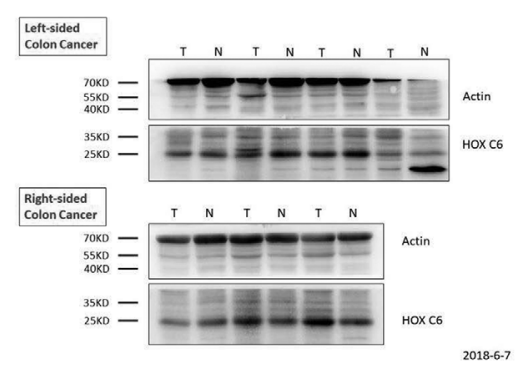

B

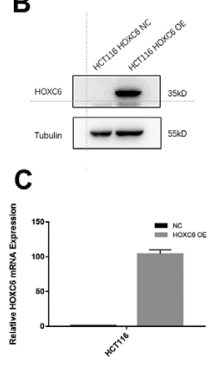

D

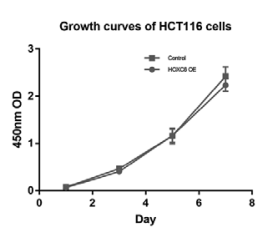

E

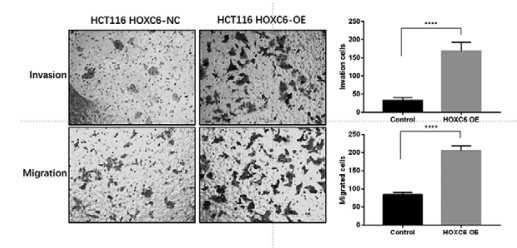

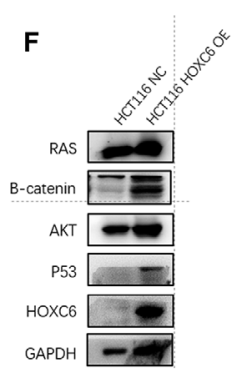
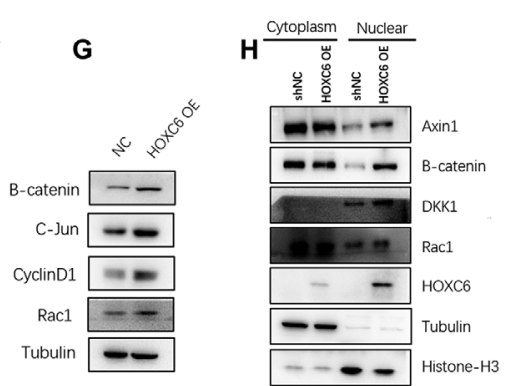

I

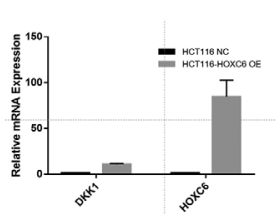

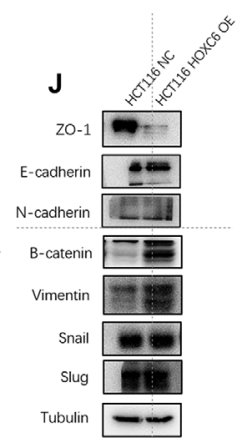

K

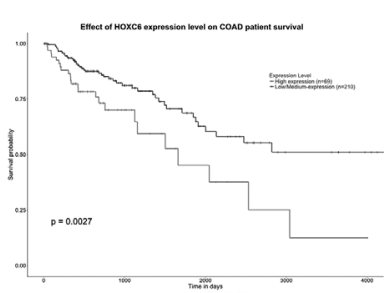

\section{Abstract IDDF2019-ABS-0341 Figure 1}

